\begin{tabular}{|c|c|c|c|}
\hline Eiszeitalter u. Gegenwart & 35 & $\begin{array}{c}181-204 \\
7 \mathrm{Abb}, 6 \mathrm{Tab} .\end{array}$ & Hannover 1985 \\
\hline
\end{tabular}

\title{
Anwendung statistischer Methoden auf Ergebnisse der Schwermineralanalyse pleistozäner Sedimente bei Nochern (Mittelrhein)
}

\author{
BARBARA WYPYRSCZYK \& WOLFGANG BOENIGK *)
}

Section, statistical methods, interpretation, heavy mineral analysis, alluvial plains, gravel, open pit, size distribution, Devonian, Tertiary, Continental Quaternary. Rhenish Schiefergebirge (Nochern Area), Rhine Valley, Nordrhein Westfalen,

TK 25: Nr. 5812

$\mathrm{K} \mathrm{u} \mathrm{r} \mathrm{z} \mathrm{f} \mathrm{a} \mathrm{s} \mathrm{s} \mathrm{u} \mathrm{n} \mathrm{g} \mathrm{:} \mathrm{Es} \mathrm{wurden} \mathrm{Schwermineraluntersuchungen} \mathrm{pleistozäner} \mathrm{Sedimente}$ am südlichen Mittelrhein bei Nochern durchgeführt.

Ziel war es, Vorstellungen über die Fehlerquellen in der Schwermineralanalyse und ihre Größenordnung zu bekommen. Dazu wurden aus einem einheitlichen Terrassenkörper entlang von 3 Profilen Proben genommen. Die Analysenergebnisse wurden statistisch aufgearbeitet. Es hat sich für die untersuchten Sedimente gezeigt, daß bei der üblicherweise geringen Probenzahl die Zählung von 100 transparenten Körnern pro Präparat ausreichend ist. Eine Verbesserung der Ergebnisse kann sinnvoll nur durch Erhöhung der Probenzahl erreicht werden.

[The Application of Statistical Methods to Results of Heavy Mineral Analysis carried out on Pleistocene Sediments in the Vicinity of Nochern (Middle Rhine)]

A b s t r a c t : Heavy mineral analysis have been carried out on pleistocene sediments at the Middle Rhine in the vicinity of Nochern.

The aim of the investigations was to get an idea of sources of error and their order in heavy mineral analysis. Samples have been taken from three pit-sections of a terrace-unit. The results of the investigations have been tested with statistical methods. From the results of the investigated sediments it can be shown that it is sufficient to count out 100 transparent grains per slide. An improvement of the results can only obtained by taking more samples.

[L'application des méthodes statistiques sur les résultats des analyses des minéraux lourds effectuées aux sédiments du Pleistocène dans la région de Nochern (Rhin Moyen)]

R é s u m é : Des analyses des minéraux lourds ont été éffectuées aux sédiments du Pleistocéne dans la région de Nochern sur le Rhin Moyen.

*) Anschrift der Autoren: Prof. Dr. W. BoenigK, Dipl.-Geol. Barbara WypyrsCZyK, Geologisches Institut der Universität zu Köln, Zülpicher Str. 49, D - 5000 Köln 1. 
Le but du présent travail était de se faire une idée des causes d'erreurs et de leur ordre de grandeur dans l'analyse des minéraux lourds. On a prélevé des échantillons de trois coupespuits d'une terrasse uniforme. Les résultats ont été discutés sur la base de différentes méthodes statistiques. Les résultats des sédiments examinés ont montré qu'il est suffisant de compter 100 grains transparents par préparation. Pour atteindre une amélioration des résultats il vaut mieux prélever plus d'échantillons.

\section{Einleitung}

Üblicherweise werden für die schwermineralanalytische Bearbeitung einer Grube entlang eines oder auch mehrerer Profile Proben genommen. Von diesen Proben wird je ein Schwermineralpräparat angefertigt und ausgezählt. Die Ergebnisse der Auszählung von $100 \mathrm{bzw} .300$ transparenten Schwermineralen je Präparat werden als repräsentativ für die gesamte Sediment-Abfolge betrachtet. Statistisch gesehen ist eine solche Aussage sehr unsicher und die Genauigkeit kaum abzuschätzen.

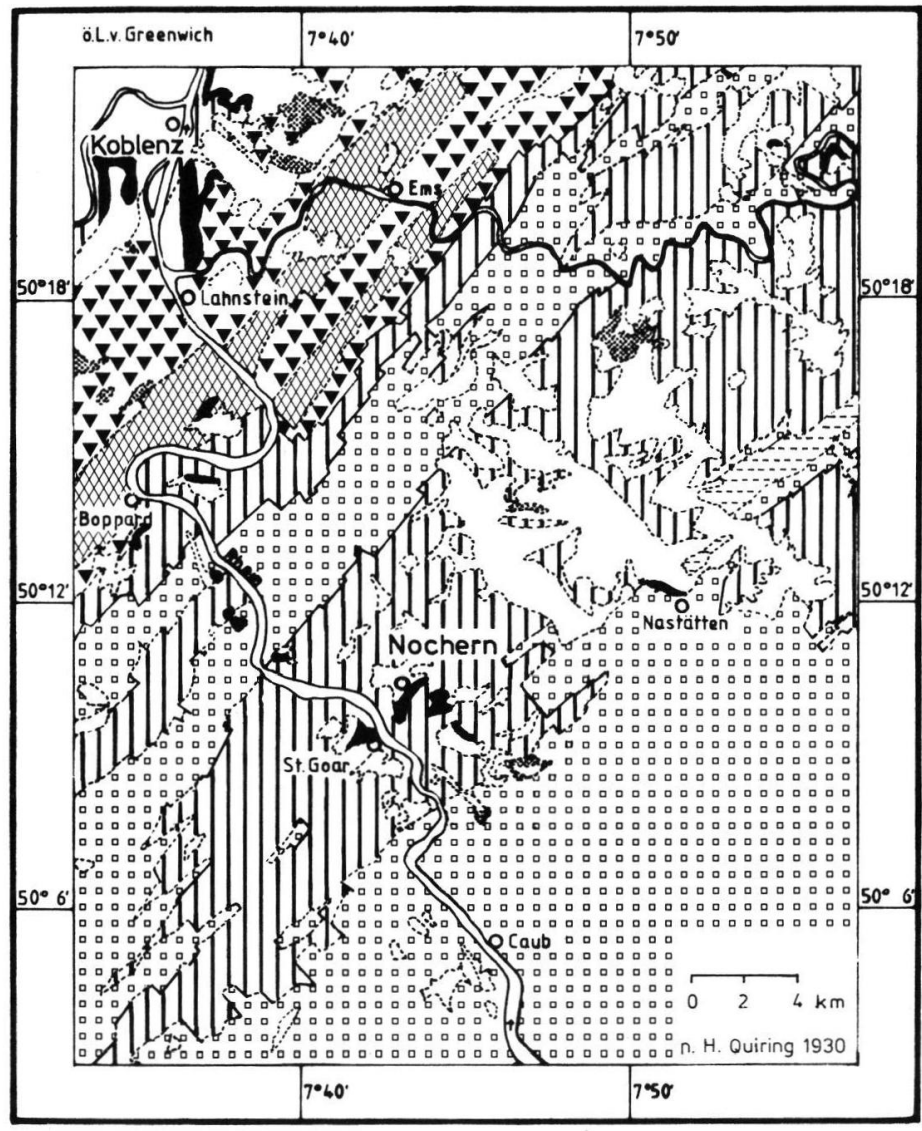

Legende

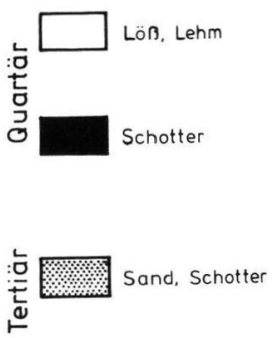

Schiefer [Eifel]

$\nabla \nabla$ Tonschiefer Sandstein [Ems]

든

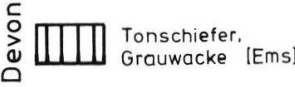

000 Hunsrückschiefer [Siegen]

Taunusquarzit [Siegen]

Abb. 1: Geologische Übersichtskarte nach H. QUIRING 1930. 
Schwermineralanalysen der beschriebenen Art werden seit Jahren durchgeführt, und es werden immer geringere Unterschiede im Mineralbestand interpretiert. Dabei wird oft nicht berücksichtigt, daß die Ergebnisse der einzelnen Schwermineralanalysen bereits infolge der heterogenen Zusammensetzung des Sedimentkörpers differieren können. Aus diesem Grund schien eine Überprüfung der Methode notwendig.

Durch Probennahme in parallel zueinander liegenden Profilen, durch das Teilen der Proben und durch das Fraktionieren dieser Teilmengen wird in der vorliegenden Arbeit ein Vergleich von Mittelwerten und Einzeldaten möglich.

Die Untersuchungen wurden durch die Deutsche Forschungsgemeinschaft finanziell unterstützt, wofür wir uns bedanken.

\section{Probennahme}

Als Test für statistische Untersuchungen der Schwermineralführung pleistozäner Rheinablagerungen wurde ein Sediment ausgewählt, das ein enges Schwermineralspektrum aufweist. Die Hauptterrassen-Schotter bei Nochern am südlichen Mittelrhein schienen für diese Untersuchungen geeignet, da man davon ausgehen kann, daß die Ablagerungen aus einer einheitlichen Schüttung stammen. Das devonische Lokalmaterial hat auf den Schwermineralgehalt der Terrassenkiese nur wenig Einfluß.

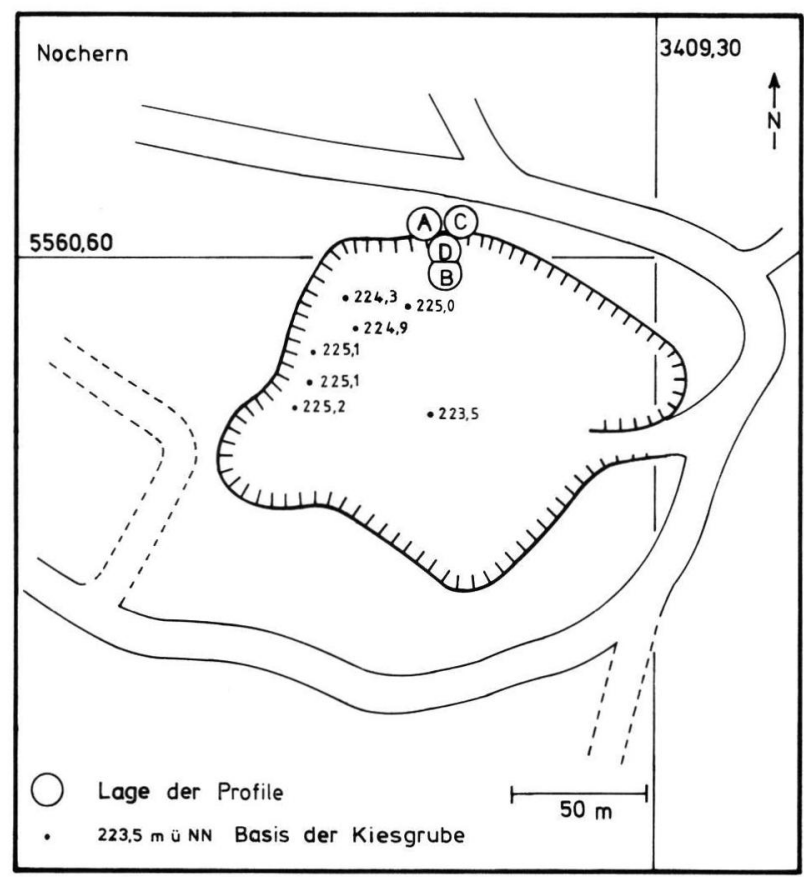

Abb. 2: Lageplan der Kiesgrube mit Eintragung der Meßpunkte und ihrer Höhenlage in $\mathrm{m}$ ü. NN. 
Ausführliche Erläuterungen zu diesem Thema finden sich bei MoRDziol (1951), SEMMEL (1972, in: Bibus \& Semmel 1977) und WyPYRSCZYK (1983).

Die Kiesgrube (H 5560,5; R 3409,2) liegt rechtsrheinisch am südlichen Mittelthein südöstlich der Ortschaft Nochern in einer Höhe von $225 \mathrm{~m}$ ü. NN (Abb. 1 u. 2). In der Grube stehen ca. 5 m mächtige kalkfreie gelbbraune Feinsande bis Grobkiese an.

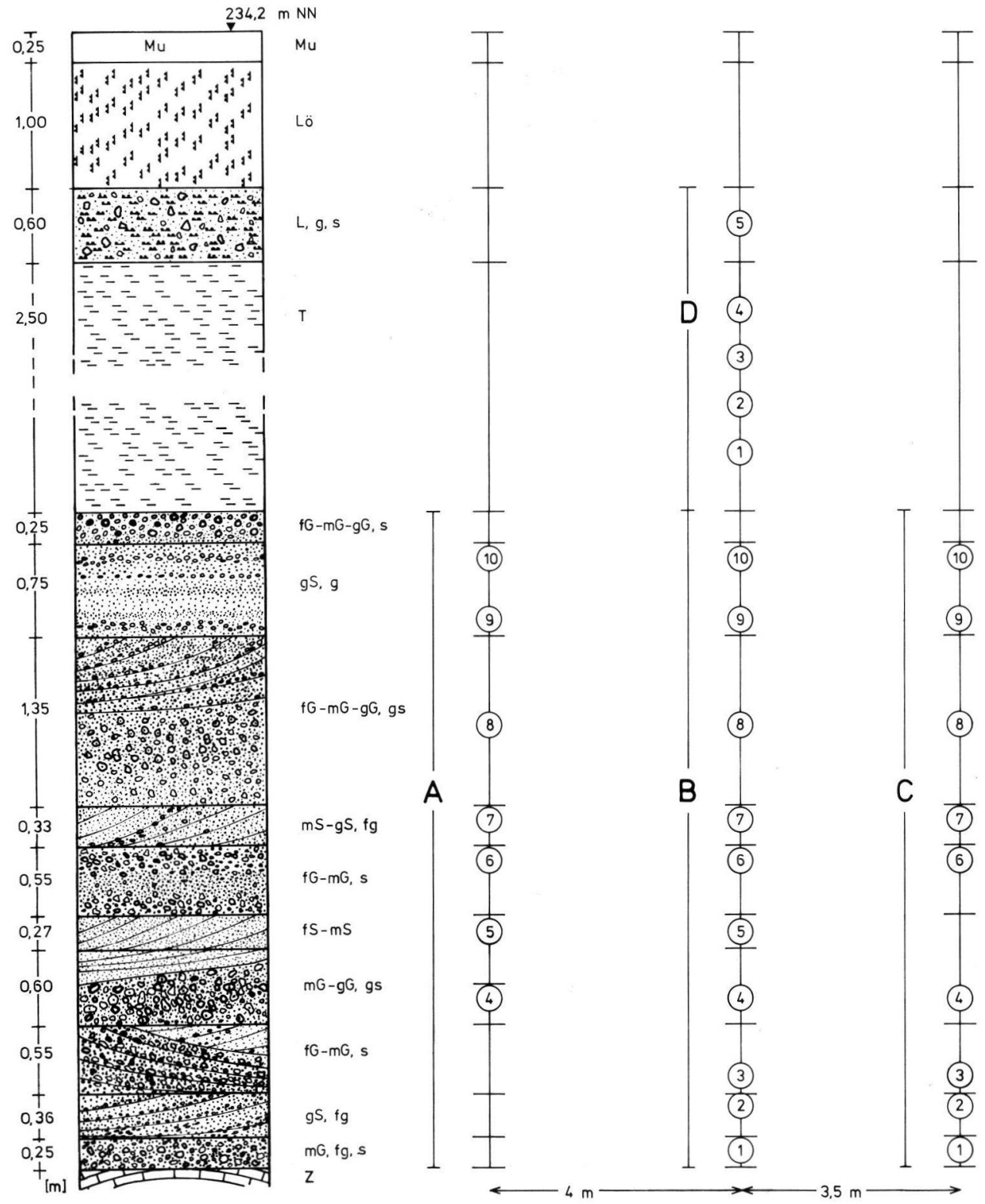

Abb. 3: Profilaufbau und Probenentnahmepunkte in den Profilen A, B, C. 
Die fluviatilen Sedimente sind in Bänke untergliedert, die in sich kreuzgeschichtet sind. Vereinzelt findet man Driftblöcke. Über den Schottern liegt 2,5 m gelber Ton; es folgt 0,6 m Solifluktionsmaterial; darüber 1,0 m Löß, auf dem sich ein 0,25 m mächtiger Boden gebildet hat (Abb. 3).

Die in der Grube bei Nochern auftretenden Schwerminerale repräsentieren das Rhein-Spektrum (RAZI RAD 1976) mit vorwiegend Epidot, Alterit, grüner Hornblende und Granat. Außerdem treten Turmalin, Zirkon, Rutil und Staurolith auf. Vereinzelt findet man Disthen, Sillimanit, Chloritoid, Glaukophan, Andalusit und Monazit. Der Gehalt an Opaken ist hoch (Tab. 1-4, im Anhang). Semmer (in: Bibus \& Semmel 1977) ordnet die quartären Sedimente bei Nochern auf Grund ihrer Höhenlage (Basis: $225 \mathrm{~m}$ ü. NN) der Hauptterrasse $t_{R 4} z u$. Dies entspricht der mittleren Hauptterrasse (mHT) von GURLITT (1949) bzw. der jüngeren Hauptterrasse (jHT) von KAISER (1961) oder nach BiRKENHAUER (1973) der Oberterrasse (OT).

Für die Probennahme wurde ein Aufschlußbereich ausgewählt, der es erlaubt, entlang von 3 parallelen Profilen jeder Schicht jeweils eine Probe zu entnehmen (Abb. 2). Die Profile liegen $3,5 \mathrm{~m}$ bzw. $4 \mathrm{~m}$ auseinander. In 4 Fällen war wegen lokaler Versturzmassen eine Entnahme von 3 Proben je Horizont nicht möglich (vgl. Abb. 3). Ergänzend wurden auch dem Tonhorizont und dem Solifluktionsmaterial Proben entnommen (Profilteil D). Der angegebene Profilaufbau (Abb. 3) ist nur für den engen Bereich $A-C$ gültig, da in die Ablagerungen zahlreiche Linsen eingeschaltet sind, die die Sediment-Abfolge bereits innerhalb des Aufschlusses ändern.

\section{Bearbeitung der Proben}

Die trockenen Proben wurden 3-geteilt. Zwei Drittel wurden in die Fraktionen $<0,063 \mathrm{~mm} ; 0,063-0,1 \mathrm{~mm} ; 0,1-0,2 \mathrm{~mm} ; 0,2-0,4 \mathrm{~mm} ; 0,4-2,0 \mathrm{~mm}$ und $>2,0 \mathrm{~mm}$ untergliedert. Aus dem dritten Teil wurde die Fraktion 0,063-0,4 mm abgesiebt. Das Gewicht der einzelnen Fraktionen wurde ermittelt. Hieraus wurden die Gewichtsanteile (in Gew. \%) unter $2,0 \mathrm{~mm}$ bzw. unter $0,4 \mathrm{~mm}$ errechnet und dargestellt (Abb. 4).

Abbildung 4a verdeutlicht die Wechsellagerung von Sanden und Schottern in der Grube Nochern. Der Anteil des sandigen Zwischenmittels in den Schotterpaketen liegt bei $50-70 \%$. Die Fraktion $0,063-0,4 \mathrm{~mm}$ ist sowohl in den Sanden als auch in den Schottern im Mittel nur mit geringen Prozentsätzen $(20-40 \%)$ vertreten. Eine Ausnahme bildet die feinsandige Schicht 5 .

Das fraktionierte Sediment wurde in Salzsäure gekocht; anschließend wurden die Schwerminerale in Bromoform mittels der Zentrifuge abgetrennt.

Zur Bestimmung des Gewichtsanteils der Schwerminerale am Sediment mußten zuvor die zur Abtrennung verwendeten Fraktionen eingewogen werden. Die Einwaage beträgt für die Fraktionen

$$
\begin{array}{ll}
0,063-0,1 \mathrm{~mm} & \text { ca. } 0,5 \mathrm{~g} \\
0,1-0,2 \mathrm{~mm} & \text { ca. } 1,5 \mathrm{~g} \\
0,2-0,4 \mathrm{~mm} & \text { ca. } 3,0 \mathrm{~g} \\
0,063-0,4 \mathrm{~mm} & \text { ca. } 3,0 \mathrm{~g}
\end{array}
$$




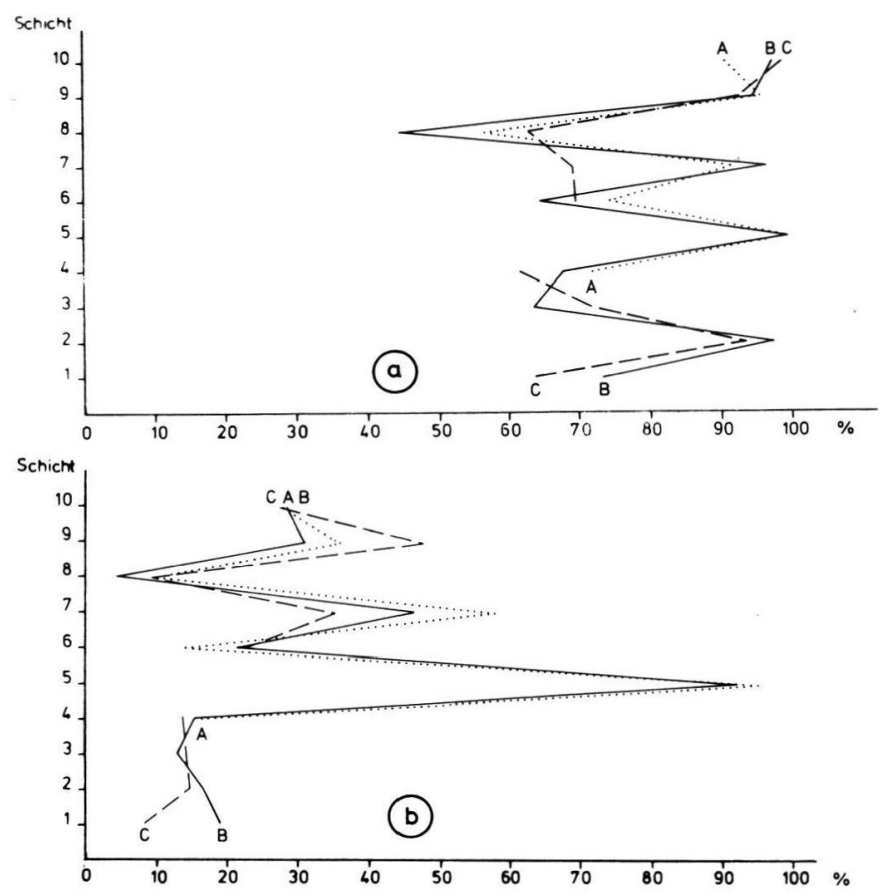

Abb. 4: Darstellung der Sedimentanteile

a) Anteil $<2,0 \mathrm{~mm}$ in den Profilen A, B, C

b) Anteil $<0,4 \mathrm{~mm}$ in den Profilen A, B, C

Die Korngrößenfraktion $0,063-0,4 \mathrm{~mm}$, aus der üblicherweise die Schwerminerale gewonnen werden, ist bei den hier vorliegenden fluviatilen Sedimenten wie folgt untergliedert:

Prozentuale Verteilung von Sediment und Schwermineralen in der Fraktion $0,063-0,4 \mathrm{~mm}(=100 \%)$.

Fraktion

Sedimentanteil (in \%) Schwermineralanteil (in \%)

$\begin{array}{lccc}0,063-0,1 & 3 & \text { ca. } 10 \\ 0,1-0,2 & 5-15 & \text { ca. } 45 \\ 0,2-0,4 & 85-95 & \text { ca. } 40\end{array}$

Diese Aufstellung zeigt, daß die Schwerminerale ungleichmäßig auf die 3 Fraktionen verteilt sind; sie sind in der Fraktion 0,1-0,2 $\mathrm{mm}$ angereichert (Abb. 5). Abb. 5 zeigt außerdem, daß der Schwermineralanteil einer Probe nicht vom Sandgehalt 


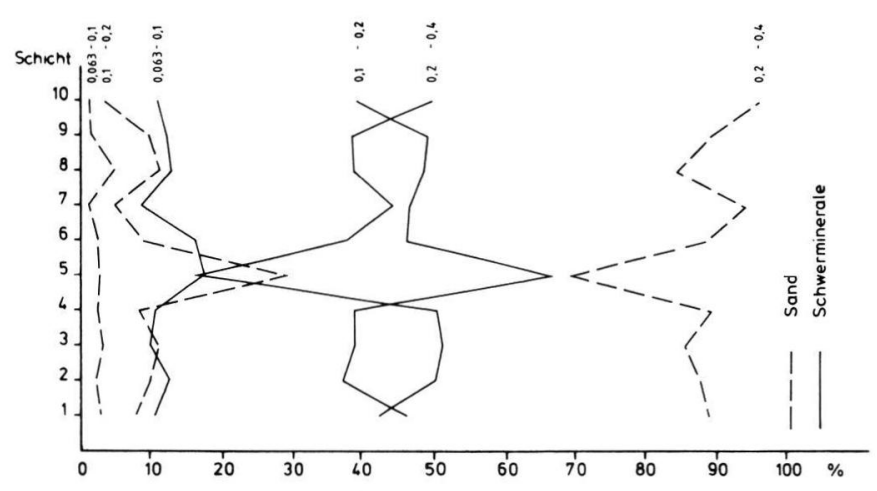

Abb. 5: Sand- und Schwermineralgehalt in Gew. \% in den Fraktionen $0,063-0,1 \mathrm{~mm} ; 0,1-0,2 \mathrm{~mm} ; 0,2-0,4 \mathrm{~mm}$; Mittelwerte aus den Ergebnissen für die Profile A, B, C.

abhängig ist, d.h. eine Probe mit geringem prozentualem Sandanteil kann einen relativ hohen Schwermineralgehalt haben.

Hinsichtlich des prozentualen Anteils der Schwerminerale in den bearbeiteten Fraktionen lassen sich keine verläßlichen Angaben machen, da sich die Einwaage bei sämtlichen Proben als zu gering erwies. Bei zu geringer Einwaage wächst die Abweichung zwischen zwei statistisch gleichen Werten infolge der Konstanz des zufälligen Fehlers enorm an (vgl. Tab. 1-4, im Anhang, Gewicht der SM in g). Die geringe Einwaage war methodisch bedingt durch die Verwendung der Zentrifuge bzw. durch einen Mangel an Material in der feinen Fraktion.

Die Scheidetrichtermethode, bei der wesentlich größere Einwaagen genommen werden, liefert reproduzierbare Werte. Man könnte auch in der Zentrifuge mit größeren Mengen arbeiten, aber die Arbeitsgeschwindigkeit würde dadurch erheblich verringert. Da $90 \%$ der Schwerminerale in der Fraktion $>0,1 \mathrm{~mm}$ liegen, bietet sich für die Abtrennung fluviatilen Materials die Scheidetrichtermethode an.

Für quantitative Untersuchungen sollte die Sedimenteinwaage für die Abtrennung in $100 \mathrm{ml}$ Bromoform $10-20 \mathrm{~g}$ betragen und die $\mathrm{zu}$ erwartende Schwermineralmenge über $0,1 \mathrm{~g}$ liegen.

Es wurden für diese Untersuchungen insgesamt 280 Schwermineralanalysen durchgeführt. Gezählt wurden entlang von parallelen Linien pro Präparat 100-150 Körner in Abhängigkeit von der Anzahl der opaken Körner, jedoch immer 100 transparente Schwermineralkörner. In 20 Präparaten wurden 300 transparente Schwerminerale pro Präparat ausgezählt.

Vergleicht man die Zählergebnisse der Fraktionen 0,063-0,1 mm; 0,1 - 0,2 mm und 0,2-0,4 mm am Beispiel des Epidot (Tab. 1-4, im Anhang), so erkennt man signifikante Unterschiede. D.h. Schwermineralanalysen für unterschiedliche Fraktionen sind quantitativ nicht miteinander zu vergleichen. 
Dagegen konnten keine qualitativen Unterschiede zwischen den Fraktionen $0,063-0,4 \mathrm{~mm}$ und $0,1-0,2 \mathrm{~mm}$ festgestellt werden, d. h. es kommt durch die Einengung des Korngrößenspektrums auf die Fraktion $0,1-0,2 \mathrm{~mm}$ nicht $\mathrm{zu}$ einem Verlust an möglichen Informationen wie KöLBL (1948) befürchtet. Diese Aussage gilt zunächst nur für die hier untersuchten Rheinsedimente.

Will man wie in der vorliegenden Arbeit die Zentrifugenmethode anwenden, so ist es zweckmäßig, sich auf die Fraktion $0,1-0,2 \mathrm{~mm}$ zu beschränken; denn die Schwerminerale sind in dieser Fraktion angereichert (Abb. 5). Zudem liegen die Schwerminerale in einer gut bestimmbaren Größe vor, und es findet eine größere Zahl an Körnern auf dem Präparat Platz; die Schwerminerale sind dadurch statistisch besser verteilt.

\section{Statistik}

Aus den oben aufgeführten Gründen wurde nur die Fraktion $0,1-0,2 \mathrm{~mm}$ mit statistischen Verfahren ausgewertet (KREYSZIG 1977; MARSAL 1967). Untersucht wurde der zufällige Fehler. Dieser ist schwierig zu fassen, denn es gibt für die Schwermineralanalyse keine Normwerte, mit denen man die empirisch gefundenen Daten vergleichen könnte.

Es treten mehrere zufällige Fehler auf. Durch die Auswahl der Probennahmepunkte wurden nur wenige Bereiche der heterogenen Ablagerung - eines Schüttungskörpers, der hinsichtlich des Liefergebietes und des Schüttungsmechanismus als eine Einheit angesehen werden muß - erfaßt. Die petrographische Zusammensetzung des Schüttungskörpers schwankt sowohl in vertikaler als auch in horizontaler Richtung um einen Mittelwert.

Ein weiterer zufälliger Fehler geht durch die Bearbeitung der Proben und durch die Herstellung der Streupräparate ein. Beim Aufbringen größerer Schwermineralmengen auf einen Objektträger muß ein Teil der Körner neben das Präparat gestreut werden, da sonst die Besetzungsdichte zu hoch wird. Wenige Körner bleiben wegen ihrer geringen Größe oder der eckigen Form in den Filtern hängen.

Die verbleibenden Schwankungen in den Analysenergebnissen werden durch die Ungenauigkeit der Zählung hervorgerufen. Der hierdurch bedingte Fehler läßt sich nicht vermeiden, aber einengen, indem man die Zahl der zu zählenden Körner erhöht.

Die Auszählung der Schwermineralpräparate erfolgt entlang von Schnittlinien. Die Auswahl dieser Schnittlinien ist zufällig und verursacht somit einen weiteren zufälligen Fehler.

Nicht eindeutig identifizierbare Minerale werden subjektiv bestimmt. So ist insbesondere die Zuordnung stark korrodierter Minerale zu den Gruppen Alterit, Quarzalterit und Alterit bis Opak unsicher. Für die Gruppe der Alterite ergeben sich beim Vergleich der Analysen verschiedener Bearbeiter große Differenzen.

Im folgenden sollen die Ergebnisse für die Fraktion $0,1-0,2 \mathrm{~mm}$ diskutiert werden (Tab. 1). 
Die Ergebnisse der Schwermineralanalyse zeigen:

a) Änderungen vom Liegenden zum Hangenden in den Profilen A, B, C.

Die Änderungen in diesen Profilen können zufällig sein oder durch Änderungen in der Materialzusammensetzung bedingt sein. Eine Erklärung für die Epidotzunahme bzw. Granat- und Hornblendeabnahme zur Oberkante der Profile liefert die Verwitterung. Die chemische Verwitterung kann den Granat und die Hornblende zersetzt haben, wodurch sich der relative Epidotanteil zwangsläufig erhöhen mußte.

b) Schwankungen innerhalb eines Horizontes.

Diese Schwankungen können sowohl zufällig sein als auch durch die heterogene Zusammensetzung des Sediments hervorgerufen worden sein. Die heterogene Zusammensetzung ist wiederum auf Sortierungseffekte innerhalb des Flußlaufs zurückzuführen.

c) Differenzen zwischen den Zählergebnissen zweier Schwermineralpräparate einer homogeniesierten Probe.

Diese Schwankungen sind zufällig, da durch die Homogenisierung alle weiteren denkbaren Einflüsse ausgeschaltet sind.

d) Schwankungen in Abhängigkeit von der Zählmenge.

Diese Schwankungen sind durch die Ungenauigkeit bedingt, mit der jede Zählung einer zufälligen Auswahl aus einer großen Menge behaftet ist. Der Fehler verringert sich mit der Erhöhung der Zahl der pro Präparat gezählten Körner und ist somit systematisch.

Der zufällige Fehler bedingt, daß die Analysen verschiedener Präparate einer Schicht, ja sogar einer homogenisierten Probe verschiedene Ergebnisse liefern. Folglich müssen auch verschiedene Bearbeiter ein und desselben Sedimentkörpers zu unterschiedlichen Ergebnissen kommen. Der zufällige Fehler ist an Hand einer Einzelprobe nicht zu erfassen. Es läßt sich nicht entscheiden, ob z. B. Unterschiede primär im Sediment vorhanden sind, oder ob sie durch die Aufbereitung der Proben, die Herstellung der Präparate oder die Zählung hervorgerufen wurden. Steht mehr als ein Präparat je Probe zur Verfügung, so sind Teilbereiche des zufälligen Fehlers erfaßbar. Es läßt sich der Fehler bestimmen, der durch die Anzahl der ausgezählten Körner pro Präparat gegeben ist.

Durch Mittelwertbildung der Daten mehrerer Schwermineraluntersuchungen einer Probe bzw. eines Horizontes werden Aussagen über die Fehler möglich, mit denen die einzelne Schwermineralanalyse behaftet ist.

\subsection{Mittelwertbildung und Berechnung der zugehörigen Standardabweichung}

Wie oben beschrieben wurden jedem Horizont 2 bzw. 3 Proben entnommen und geteilt. Da man von einer einheitlichen Schüttung ausgehen kann, muß die Schwer- 
mineralverteilung in den Proben aus einem Horizont statistisch gesehen gleich sein. Somit müssen die Differenzen zwischen den empirisch ermittelten Werten durch zufällige Fehler verursacht sein. Es ist daher zulässig, die aus diesen Proben gewonnenen Zählergebnisse zu mitteln und, da der Probenumfang mit 4-6 Proben je Horizont sehr gering ist und der Erwartungswert unbekannt ist, die Stichprobenstandardabweichung zu berechnen.

Die Epidotgruppe ist in den Streupräparaten am stärksten vertreten (Tab. 1-4, im Anhang). Mittelwerte und Standardabweichungen der Epidotgruppe wurden aufgetragen (Abb. 6).

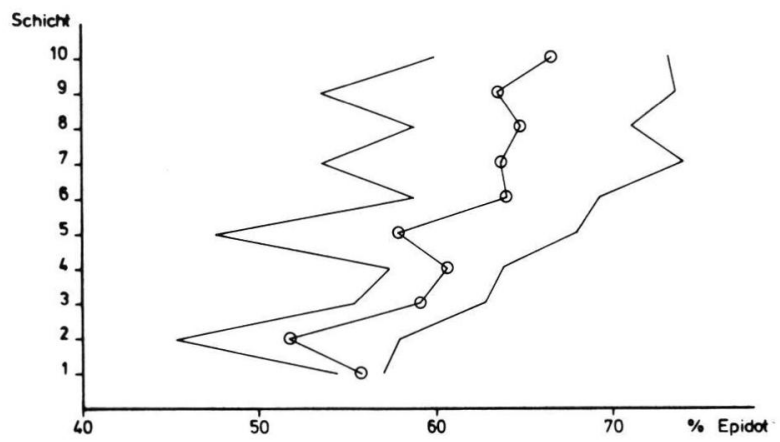

Abb. 6: Mittelwerte und Standardabweichungen für den Epidotanteil des Sediments in der Fraktion 0,1-0,2 mm.

Die Mittelwerte zeigen vom Liegenden zum Hangenden eine leichte Zunahme des Epidotanteils. Es lag nahe zu überprüfen, ob sich diese Tendenz auch separat in den Profilen A, B und C zeigt (Abb. 7). Außerdem wurden die Daten betrachtet, die sich für Profil B aus der Zählung von 300 transparenten Schwermineralen je Präparat ergaben.

Die Verteilung innerhalb der Profile A, B und C kann die Annahme, daß der Epidotgehalt vom Liegenden zum Hangenden ansteigt, nicht stützen. Betrachtet man die Darstellung für Profil A (Abb. 7), so muß man auf eine Gleichverteilung schließen, bei der die Abweichungen der empirisch gefundenen Werte von einem einheitlichen Mittelwert als zufällig angesehen werden. Dagegen zeigt die Darstellung für Profil C (Abb. 7) ähnlich den Mittelwerten aus den Profilen A, B und C einen Anstieg des Epidotanteils um $20 \%$. Die Darstellung für Profil B (Abb. 7) zeigt einen geringfügigen Anstieg des Epidotanteils. Die große Schwankungsbreite der Werte erlaubt es aber auch, an Hand dieses Profils auf eine Gleichverteilung zu schließen. Eindeutigere Ergebnisse für Profil B ergeben sich aus der Zählung von 300 Körnern pro Präparat auf Grund der Einengung der Schwankungsbreite (Abb. 7). Die Darstellung des Epidotanteils zeigt jetzt Gleichverteilung. Nur in Schicht 5 erreicht der Epidotgehalt höhere Werte. Die Verteilung der Epidotgehalte in den Profilen A, B und C divergiert stark. Isoliert betrachtet, führen die Ergebnisse für diese Profile zu unterschiedlichen Interpretationen. 

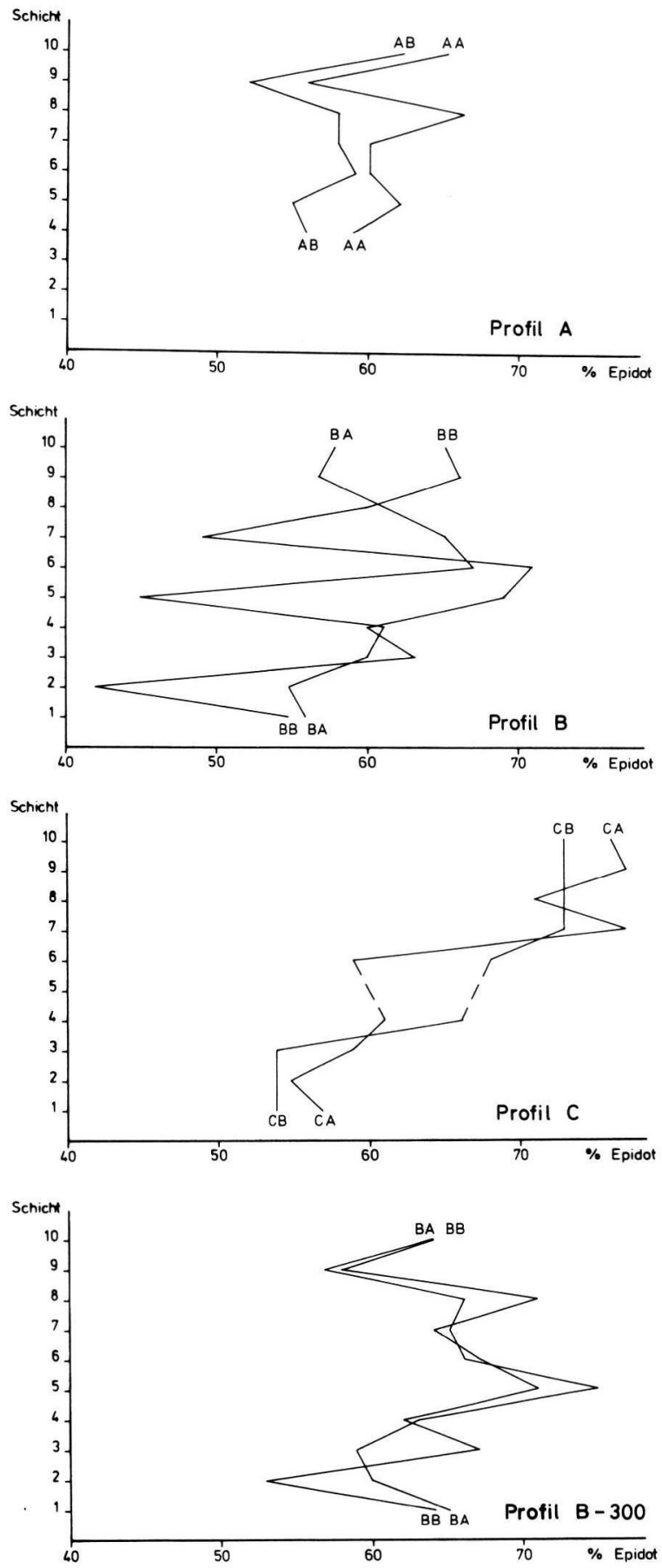

Abb. 7: Verteilung des Epidotanteils der Fraktion 0,1-0,2 mm in den Profilen A, B, C;

Profil B-300 auf der Basis von 300 transparenten Schwermineralkörnern. 
Die bisherigen Untersuchungen ergaben kein eindeutiges Ergebnis. Mit Hilfe statistischer Tests $(4.2-4.7)$ wurde überprüft, ob eine Gleichverteilung vorliegt (Nullhypothese).

\subsection{Vergleich der Horizonte; Varianzanalyse}

Zum Vergleich der Epidotverteilung in verschiedenen Horizonten ist die Varianzanalyse geeignet. Sie vergleicht die Variationen innerhalb einer Schicht mit den Variationen, die sich von Schicht zu Schicht ergeben. Die Varianzanalysen bestätigen die aus den Darstellungen (Abb. 7) hervorgehenden Tendenzen. In den Profilen A und B liegen keine signifikanten Unterschiede zwischen den Ergebnissen für die einzelnen Horizonte vor; die Zählergebnisse für eine Probe schwanken in der gleichen Größenordnung wie die Zählergebnisse für Proben aus verschiedenen Horizonten. Die Nullhypothese wird angenommen. Die Ergebnisse besagen, daß der Schotterkörper zwar heterogen ist, aber einen einheitlichen Schüttungskörper mit einem einheitlichen Liefergebiet darstellt.

Die Ergebnisse für Profil C sprechen gegen die Nullhypothese. Daher wurden für Profil C die Konfidenzintervalle berechnet. Diese überlappen sich. Die Tendenz nach Abb. 7, die auch durch die Varianzanalyse bestätigt wird, wird damit in Frage gestellt. Eine eindeutige Aussage ist bei nur 2 Präparaten je Probe und je Horizont auch bei Anwendung statistischer Tests nicht möglich.

\subsection{Vergleich der Profile A, B, C}

Vergleicht man die Profile A, B, C mittels statistischer Tests, so erkennt man, daß sämtliche Proben einer Grundgesamtheit angehören können. Zwar zeigen Varianzanalyse und t-Test, daß die Unterschiede zwischen den Profilen signifikant sind, doch die Überlappung der Konfidenzintervalle spricht dagegen.

\subsection{Vergleich der Horizonte auf der Basis der Ergebnisse für die Profile A, B und C}

Geht man davon aus, daß die in gleicher Höhe entnommenen Proben jeweils der gleichen Grundgesamtheit entnommen sind (4.3), so ist es zulässig, die Horizonte auf der Basis der Ergebnisse für die Profile A, B und C miteinander zu vergleichen (Varianzanalyse). Die Nullhypothese, daß die Proben aller Horizonte einer Grundgesamtheit angehören, wird angenommen. Der Anstieg des Epidotanteils (Abb. 6) kann nach Anwendung der statistischen Tests als zufällig angesehen werden. Die Betrachtungen für die bisherigen Ergebnisse für die Profile A, B und C sowie für die Mittelwerte aus diesen Profilen machen eine signifikante Änderung vom Liegenden zum Hangenden unwahrscheinlich. 


\subsection{Vergleich der Zählung von 100 mit der Zählung von 300 transparenten Schwermineralen je Präparat}

Die Mittelwerte für eine Probe, gebildet auf der Basis von 2 Zählungen mit je 100 transparenten Schwermineralkörnern, haben enorme Konfidenzintervalle. Es stellt sich die Frage, ob zwei Präparate einer Probe tatsächlich derart stark differieren, oder ob die Unterschiede durch die Auszählung von nur 100 transparenten Schwermineralkörnern je Präparat vorgetäuscht werden.

Die Varianzanalyse prüft, ob auch bei Zählung von 300 Körnern sämtliche Horizonte einer Grundgesamtheit angehören können (Nullhypothese). Für die Ergebnisse der Zählung von 100 Körnern wird die Nullhypothese angenommen (4.2); für die Zählung von 300 Körnern wird sie verworfen. Hier kommt zum Ausdruck, daß die Zählung von 300 Körnern nur eine Einengung der Schwankungsbreite in einem Horizont bewirkt. Hingegen bleiben die Schwankungen in der Vertikalen bestehen. Auch die maximale Abweichung vom Mittelwert wird nicht verringert.

Es wurden zusätzlich die Konfidenzintervalle berechnet. Diese überlappen sich sowohl bei der Zählung von 100 als auch bei der Zählung von 300 Körnern je Präparat. Danach ist es möglich, daß auch bei Zählung von 300 Körnern je Präparat sämtliche wahren Werte in den Überlappungsbereich fallen und nicht signifikant differieren.

\subsection{Schwankungen zwischen den Ergebnissen}

für 2 Schwermineralpräparate einer homogenisierten Probe

Statistisch gesehen muß die Schwermineralverteilung in 2 Präparaten einer homogenisierten Probe gleich sein. Der Erwartungswert für die Differenz der Ergebnisse ist folglich Null. Die empirisch ermittelten Differenzen zwischen den Ergebnissen von jeweils 2 Präparaten einer Probe sind Zufallsvariable.

Den folgenden Berechnungen liegen die 52 Präparate aus den Profilen A, B und C und 18 weitere Präparate aus 9 Proben derselben Grube zu Grunde. Es ergeben sich je Fraktion 35 Differenzen zwischen 2 statistisch gesehen gleichen Präparaten.

Aus den Standardabweichungen zu den jeweils 35 Werten wurden die Grenzen errechnet, innerhalb derer $95 \%, 90 \%$ und $80 \%$ aller möglichen Ergebnisse liegen können. Die Grenzen liegen um folgende Werte auseinander:

\begin{tabular}{cccccr} 
Epidotgruppe & Fraktion & Mittelwert & \multicolumn{3}{c}{ Wahrscheinlichkeit } \\
& & & $95 \%$ & $90 \%$ & $80 \%$ \\
& $0,063-0,4 \mathrm{~mm}$ & $51,7 \pm 11,1$ & 25,4 & 21,3 & 16,1 \\
& $0,063-0,1 \mathrm{~mm}$ & $58,8 \pm 6,8$ & 14,7 & 12,3 & 9,6 \\
$0,1-0,2 \mathrm{~mm}$ & $60,9 \pm 7,7$ & 15,4 & 13,0 & 10,1 \\
& $0,2-0,4 \mathrm{~mm}$ & $35,8 \pm 16,6$ & 31,1 & 26,1 & 20,3
\end{tabular}


Berechnung für Profil B, Fraktion 0,1-0,2 mm für die Ergebnisse der Auszählung von 100 und 300 transparenten Schwermineralkörnern je Präparat:

\begin{tabular}{|c|c|c|c|c|c|}
\hline \multirow[t]{2}{*}{ Epidotgruppe } & \multirow[t]{2}{*}{ Zählmenge } & \multirow[t]{2}{*}{ Mittelwert } & \multicolumn{3}{|c|}{ Wahrscheinlichkeit } \\
\hline & & & $95 \%$ & $90 \%$ & $80 \%$ \\
\hline & 100 & $59,3 \pm 7,8$ & 22,3 & 18,7 & 14,6 \\
\hline & 300 & $64,1 \pm 5,2$ & 8,1 & 6,8 & 5,3 \\
\hline
\end{tabular}

Jede dieser Berechnungen geht von einem empirisch gefundenen Mittelwert aus und kann daher auch nur für diesen Mittelwert einschließlich eines Toleranzbereiches gelten. Für andere Mittelwerte müssen neue Berechnungen durchgeführt werden.

Halbiert man die Werte, die sich für die maximale Differenz zwischen 2 unabhängigen Zählungen einer homogenisierten Probe ergeben, so erhält man die maximal möglichen Abweichungen vom Mittelwert bei einer Wahrscheinlichkeit von 95 \%, 90 \% und $80 \%$. Die Abweichung kann positiv oder negativ sein.

Die Zählung von 300 Schwermineralen je Präparat wurde für Profil B in der Fraktion $0,1-0,2 \mathrm{~mm}$ durchgeführt. Diese Auszählung verengt die Grenzen, innerhalb derer $95 \%, 90 \%$ und $80 \%$ aller möglichen Werte liegen. Nach KölBL $(1948,54)$ erhöht sich durch mehrfaches Auszählen eines Präparates nur die Genauigkeit für dieses eine Präparat. Dies gilt auch, wenn statt 100 jetzt 300 Schwermineralkörner je Präparat ausgezählt werden. $\mathrm{Da}$ ein Schüttungskörper stets heterogen ist, kann die höhere Zählgenauigkeit für eine Probe nicht geeignet sein, die gesamte Grube zu charakterisieren. Die Fehlergrenzen für eine Probe sollten nicht enger sein als die Fehlergrenzen, die durch die Schwankungsbreite im Sediment vorgegeben sind.

Die Fehlergrenzen werden ebenfalls verengt, wenn den Berechnungen eine große Zahl von Proben zu Grunde liegt; denn durch die Erhöhung der Probenzahl verringert sich die Standardabweichung für die Differenz zwischen 2 unabhängigen Zählergebnissen für eine Probe.

Einen Überblick über die Schwermineralverteilung in einem einheitlichen Sedimentkörper erhält man am ehesten, wenn man möglichst viele Proben untersucht und aus den Ergebnissen Mittelwerte bildet. Dadurch erfaßt man ein breites Spektrum der Ablagerung und grenzt zudem das Konfidenzintervall für den Mittelwert ein. Diese Eingrenzung ist sicher realistischer als die Eingrenzung, die sich durch die höhere Zählgenauigkeit bei einem Präparat ergibt.

\subsection{Schwankungen des Epidotanteils in der Grube}

$\mathrm{Da}$ die Ergebnisse für die Schwankungen des Epidotanteils durch die Addition mehrerer unabhängiger Zufallsgrößen hervorgerufen werden, müssen sich die Varianzen der einzelnen Zufallsgrößen nach dem Fehlerfortpflanzungsgesetz von GAUSS zu der Varianz sämtlicher auftretenden Größen summieren. Die Varianz einer Summe von 2 Zufallsgrößen ist gleich der Summe der Varianzen der beiden Zufallsgrößen: 
$\delta_{1+2}^{2}=\delta_{1}^{2}+\delta_{2}^{2}$. Die Varianz der Summe $\delta_{1+2}^{2}$ ergibt sich im vorliegenden Fall aus den Zählergebnissen. Die Varianz der Zählung $\delta_{2}^{2}$ ergibt sich rechnerisch aus der Binomialverteilung nach der Formel: $\delta^{2}=\mathrm{n} p(1-\mathrm{p})$ mit $\mathrm{n}=$ Anzahl der ausgezählten Körner je Präparat; $p=$ Wahrscheinlichkeit für das Eintreffen eines bestimmten Ereignisses.

Durch die Bildung der Differenz $\delta_{1+2}^{2}-\delta_{2}^{2}$ erhält man einen Wert, der die Schwankungen des Epidotanteils in der Grube und den Bearbeitungsfehler beinhaltet $\left(\delta_{1}\right)$.

$\delta_{1+2}=7,76$

Standardabweichung der Summe

$\delta_{2}=4,87$ Zählfehler für $\mathrm{n}=100$

$\delta_{1}^{2}=7,76^{2}-4,87^{2}$

$\delta_{1}=6,04$

Da die Summe $\delta_{1}$ von Bearbeitungsfehler und den Schwankungen des Epidotanteils in der Grube in diesem Beispiel größer ist als der Zählfehler $\delta_{2}$, kann eine Verbesserung des Ergebnisses besser durch Herabsetzung des Fehlers $\delta_{1}$ (Bearbeitungsfehler plus Schwankungen in der Grube) als durch eine Verminderung des Zählfehlers $\delta_{2}$ erreicht werden. Die Herabsetzung des Fehlers $\delta_{1}$ muß durch Erhöhung der Probenzahl erfolgen. Durch die Bearbeitung einer großen Anzahl Proben kommt man zu realistischeren Ergebnissen als durch das sehr genaue Auszählen einzelner Präparate. Dies gilt um so mehr, als in diesem Beispiel die Probenzahl sehr viel höher liegt als bei dem üblichen Bearbeitungsverfahren. Es wurde die 3-fache Probenzahl genommen und jede Probe noch einmal geteilt.

\section{Zusammenfassung}

Üblicherweise werden für die schwermineralanalytische Bearbeitung entlang ausgewählter Profile Proben genommen. Von diesen Proben wird je ein Schwermineralpräparat angefertigt und ausgezählt. Das Ergebnis der Auszählung von 100 transparenten Schwermineralen je Präparat wird als repräsentativ für eine Sediment-Abfolge angesehen.

Statistisch gesehen ist eine solche Aussage sehr unsicher. Es ist kaum möglich, die Genauigkeit solcher Schwermineralanalysen abzuschätzen. Durch Probennahme in parallel zueinander liegenden Profilen, durch das Teilen der Proben und das Fraktionieren dieser Teilmengen war die Möglichkeit zur Berechnung von Mittelwerten und ihren Standardabweichungen sowie zum Vergleich der Einzeldaten gegeben. Die Ergebnisse lassen Rückschlüsse auf bisherige Schwermineralanalysen zu.

Wie die Untersuchungen zeigten, kann durch eine zu geringe Probenzahl je Aufschluß eine Änderung im Schwermineralgehalt (Abb. 7) vorgetäuscht werden. Dies führt zu Fehlschlüssen, die durch die Ergebnisse verschiedener statistischer Tests noch bestärkt werden.

Da selbst in dem vorliegenden Beispiel der Zählfelder der kleinere Fehler ist, erscheint eine Zählung von 100 transparenten Schwermineralen je Präparat in jedem 
Fall ausreichend. Einen Überblick über die Schwermineralverteilung erhält man am sichersten, wenn man eine möglichst hohe Probenzahl untersucht und aus den Ergebnissen Mittelwerte bildet.

Da ein Schüttungskörper stets heterogen ist, kann eine höhere Zählmenge (z. B. 300 Körner) je Präparat nicht geeignet sein, die Schwermineralpopulation einer Grube genauer zu charakterisieren. Die Fehlergrenzen für eine Probe sollten nicht enger sein als die Fehlergrenzen, die durch die natürliche Schwankungsbreite im Sediment vorgegeben sind.

Die Überlegungen bezüglich der quantitativen Untersuchungen gelten nur für Sedimente, die den untersuchten zumindest ähnlich sind - also für fluviatile Sande und Schotter des Pleistozäns mit einem Einzugsgebiet wie dem des Mittelrheins zur Hauptterrassenzeit. Bereits die Mittelterrassen haben eine andere Schwermineralverteilung.

\section{Schriftenverzeichnis}

Bibus, E. \& Semmel, A. (1977): Über die Auswirkung quartärer Tektonik auf die altpleistozänen Mittelrhein-Terrassen. - Catena, 4: 385-408, 4 Fig., 1 Tab.; Gießen.

Birkenhauer, J. (1973): Zur Chronologie, Genese und Tektonik der plio-pleistozänen Terrassen am Mittelrhein und seinen Nebenflüssen. - Z. Geomorph. N. F., 17(4): 489-498, 1 Tab.; Berlin, Stuttgart.

Gurutr, D. (1949): Das Mittelrheintal. Formen und Gestalt. — Forsch. dt. Lkde., 46: 159 S., 28 Abb., 2 Taf.; Stuttgart.

KAISER, K. (1961): Gliederung und Formenschatz des Pliozäns und Quartärs am Mittel- und Niederrhein, sowie in den angrenzenden Niederlanden unter besonderer Berücksichtigung der Rheinterrassen. - Köln u. Rheinld., Festschrift, 33: 236-278, 6 Abb., 7 Tab., $3 \mathrm{Kt}$; Wiesbaden.

KörbL, L. (1948): Untersuchungen über die Verteilung von Schwermineralien im niederbayrischen Tertiär. - Neues Jb. Min., Geol., Paläont., 79A: 39-103, 13 Abb.; Stuttgart.

KreYZig, E. (1977): Statistische Methoden und ihre Anwendungen, 6 Aufl. - 451 S., 82 Abb.; Göttingen (Vandenhoeck \& Ruprecht).

Marsal, D. (1967): Statistische Methoden für Erdwissenschaftler. — 152 S., 40 Abb.; Stuttgart (Schweizerbart).

Mordziol, C. (1951): Der geologische Werdegang des Mittelrheintales. - Geol. d. Mittelrheintales u. d. Eifel, 1: 76 S., 38 Abb., 6 Tab.; Melle.

RAZI RAD, M. (1976): Schwermineraluntersuchungen zur Quartär-Stratigraphie am Mittelrhein. - Sonderveröff. Geol. Inst. Univ. Köln, 28: 164 S., 51 Abb., 6 Tab., 1 Beil.; Köln.

Semmel, A. (1972): Fragen der Quartärstratigraphie im Mittel- und Oberrhein-Gebiet. - Jber. u. Mitt. Oberrh. geol. Ver., N. F., 54: 61-71, 2 Abb.; Frankfurt a.M. 
SINDOwsKI, K.-H. (1938): Grundsätzliches zur Schwermineralanalyse der diluvialen Rheinterrassen und Lösse des Mittel- und Niederrheingebietes. - Z. dt. geol. Ges., 92: 477-499, $6 \mathrm{Abb}$; Stuttgart.

WYPYRSCZYK, B. (1983): Statistische Untersuchungen der Schwermineralführung pleistozäner Sedimente am Mittelrhein. - Dipl.-Arb., Geol. Inst. Univ. Köln: 134 S., 11 Abb., 9 Tab.; Köln. - [Unveröff.]

Manuskript eingegangen am 19. 6. 1984, Nachträge März 1985.

\section{Anhang}

Tabellen $1-6$

Erläuterung zu den Tabellen $1-4$ (Probennummer):

Beispiel: B $1 \mathrm{Ac}$

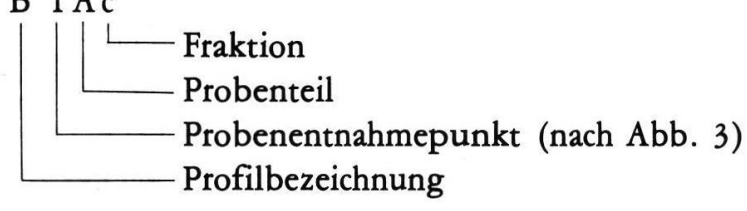


Tab. 1: Schwermineralanalysen in der Fraktion 0,1-0,2 $\mathrm{mm}$ für Profil B auf der Basis von 100 und 300 (Profil B-300) transparenten Körnern.
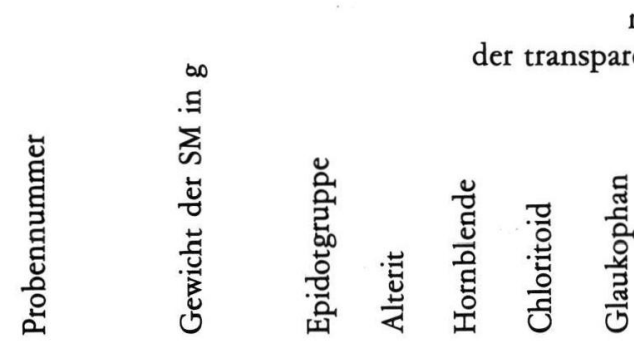

relative Korn \%

arenten Schwermineralkörner

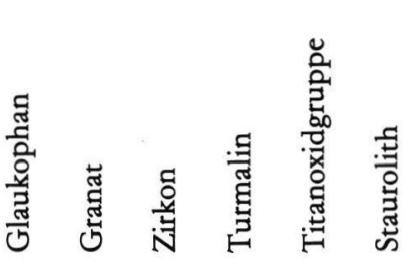

relative Korn \% aller gezählten Körner

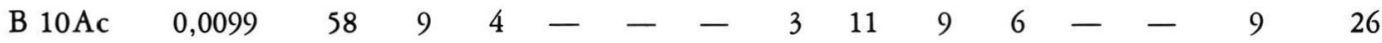

$\begin{array}{lllllllllllllllll}\text { B } 10 \mathrm{Bc} & 0,0133 & 65 & 11 & 1 & 1 & - & 1 & - & 6 & 3 & 11 & 1 & - & 3 & 26\end{array}$

$\begin{array}{lllllllllllllllll}\text { В } 9 \text { Ac } & 0,0155 & 57 & 6 & 5 & - & - & - & 4 & 17 & 2 & 5 & 4 & - & 7 & 25\end{array}$

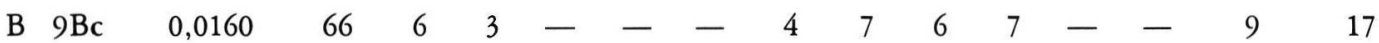

$\begin{array}{lllllllllllllllllllll}\text { B } & 8 \mathrm{Ac} & 0,0067 & 61 & 14 & 7 & 1 & - & 2 & 3 & 6 & 2 & 4 & - & - & 5 & 22\end{array}$

$\begin{array}{llllllllllllllllll}\mathrm{B} & 8 \mathrm{Bc} & 0,0087 & 60 & 17 & 2 & 1 & - & 2 & 2 & 6 & 3 & 4 & 1 & - & 9 & 12\end{array}$

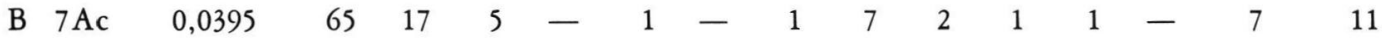

$\begin{array}{lllllllllllllllll}\text { В } & 7 \mathrm{Bc} & 0,0408 & 49 & 19 & 10 & - & - & 6 & 3 & 2 & 2 & 5 & 4 & - & 9 & 19\end{array}$

В 6 Ac $0,0080 \quad 67 \quad 12 \quad 5 \quad-\quad \begin{array}{llllllllllllllll} & 5 & 5 & 3 & 3 & 2 & - & - & 5 & 15\end{array}$

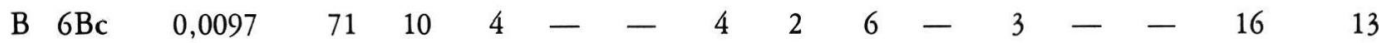

$\begin{array}{llllllllllllllllll}\mathrm{B} & 5 \mathrm{Ac} & 0,0162 & 45 & 35 & 2 & 2 & - & 2 & 1 & 1 & - & 5 & - & & \end{array}$

$\begin{array}{llllllllllllllllll}\text { B } & 5 \mathrm{Bc} & 0,0128 & 69 & 17 & 1 & - & - & 1 & 3 & 4 & 1 & 3 & 1 & - & 12 & 10\end{array}$

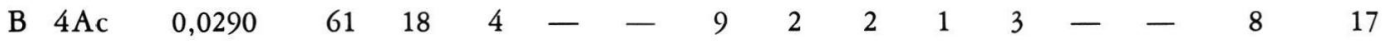

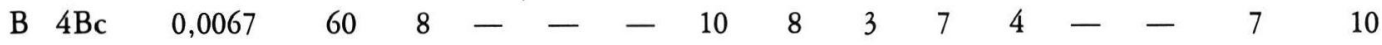

$\begin{array}{lllllllllllllllll}\text { B } & 3 \mathrm{Ac} & 0,0277 & 60 & 13 & 6 & 3 & - & 6 & 1 & 4 & 2 & 4 & 1 & - & 10 & 18\end{array}$

$\begin{array}{llllllllllllllllllll}\text { В } & 3 \mathrm{Bc} & 0,0280 & 63 & 19 & 5 & - & - & 5 & 3 & 2 & 2 & 1 & - & - & 5 & 12\end{array}$

$\begin{array}{llllllllllllllllll}\mathrm{B} & 2 \mathrm{Ac} & 0,0165 & 55 & 19 & 10 & - & - & 4 & 1 & 5 & 2 & 3 & 1 & - & 10 & 14\end{array}$

$\begin{array}{lllllllllllllllll}\text { B } & 2 \mathrm{Bc} & 0,0169 & 42 & 26 & 16 & - & 1 & 5 & 2 & 1 & 4 & 1 & 2 & - & 6 & 10\end{array}$

$\begin{array}{lllllllllllllllll}\text { B } & 1 \text { Ac } & 0,0409 & 56 & 25 & 2 & - & - & 5 & 2 & 5 & 1 & 2 & 2 & - & 3 & 19\end{array}$

$\begin{array}{lllllllllllllllllll}\text { B } & 1 \mathrm{Bc} & 0,0351 & 55 & 21 & 5 & - & - & 8 & 1 & 5 & 5 & - & - & - & 9 & 9\end{array}$ 
Profil B-300

Fortsetzung Tab. 1

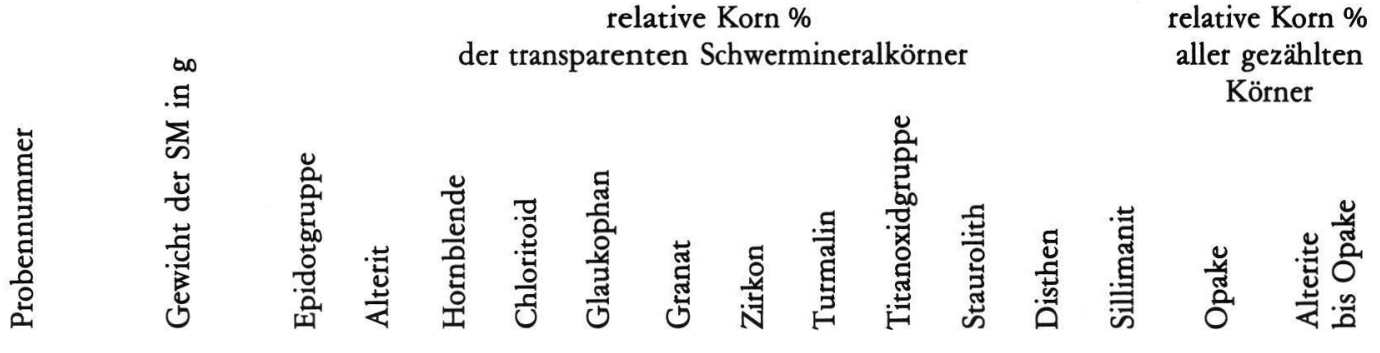

$\begin{array}{llllllllllllllll}\text { B } 10 \mathrm{Ac} & 0,0099 & 64 & 6 & 4 & \mathrm{x} & - & 1 & 3 & 10 & 4 & 4 & 2 & 1 & 8 & 28\end{array}$

$\begin{array}{llllllllllllllll}\text { B } 10 \mathrm{Bc} & 0,0133 & 64 & 8 & 3 & 1 & - & 3 & 1 & 8 & 2 & 7 & 2 & 1 & 7 & 27\end{array}$

$\begin{array}{llllllllllllllllll}\text { B } 9 \text { Ac } & 0,0155 & 57 & 7 & 2 & \text { x } & \text { x } & 1 & 3 & 15 & 3 & 8 & 3 & - & 8 & 26\end{array}$

$\begin{array}{lllllllllllllllllll}\text { B } & 9 \mathrm{Bc} & 0,0160 & 58 & 7 & 4 & 1 & - & 2 & 1 & 13 & 4 & 7 & 3 & - & 8 & 28\end{array}$

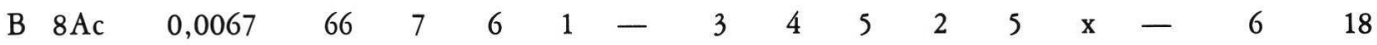

$\begin{array}{llllllllllllllllll}\mathrm{B} & 8 \mathrm{Bc} & 0,0087 & 71 & 12 & 2 & 1 & - & 3 & 3 & 4 & 1 & 2 & \mathrm{x} & 1 & 6 & 20\end{array}$

$\begin{array}{lllllllllllllllll}\text { B } & 7 \mathrm{Ac} & 0,0395 & 65 & 14 & 4 & \mathrm{x} & - & 2 & 1 & 3 & 4 & 4 & 2 & - & 9 & 17\end{array}$

$\begin{array}{lllllllllllllllll}\text { В } & 7 \mathrm{BC} & 0,0408 & 64 & 13 & 5 & 1 & - & 3 & 3 & 6 & 2 & 2 & 1 & - & 7 & 18\end{array}$

$\begin{array}{lllllllllllllllll}\text { B } & 6 \mathrm{Ac} & 0,0080 & 66 & 11 & 6 & \mathrm{x} & \mathrm{x} & 2 & 1 & 7 & 2 & 3 & 1 & - & 7 & 22\end{array}$

$\begin{array}{lllllllllllllllll}\text { В } & 6 \mathrm{Bc} & 0,0097 & 67 & 14 & 3 & - & - & 4 & 2 & 5 & 2 & 2 & 1 & - & 6 & 19\end{array}$

$\begin{array}{lllllllllllllllll}\text { B } & 5 \mathrm{Ac} & 0,0162 & 75 & 11 & 2 & - & - & 3 & 1 & 4 & 1 & 3 & - & \mathbf{x} & 6 & 23\end{array}$

$\begin{array}{lllllllllllllllll}\text { B } & 5 \mathrm{BC} & 0,0128 & 72 & 15 & \mathrm{x} & 1 & - & 1 & 2 & 4 & 1 & 3 & 1 & - & 8 & 22\end{array}$

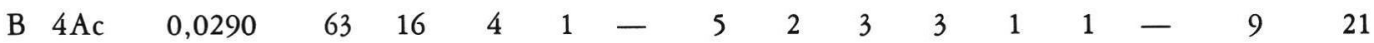

$\begin{array}{lllllllllllllllll}\text { В } & 4 \mathrm{Bc} & 0,0067 & 62 & 11 & 1 & \mathrm{x} & - & 10 & 5 & 2 & 4 & 4 & \mathrm{x} & - & 9 & 8\end{array}$

$\begin{array}{lllllllllllllllll}\text { В } & 3 \mathrm{AC} & 0,0277 & 59 & 16 & 5 & 2 & - & 7 & 2 & 6 & 1 & 2 & \mathrm{x} & \mathrm{x} & 6 & 18\end{array}$

$\begin{array}{llllllllllllllllll}\text { В } & 3 \mathrm{Bc} & 0,0280 & 67 & 12 & 3 & 1 & - & 6 & 2 & 4 & 1 & 3 & 1 & - & 10 & 13\end{array}$

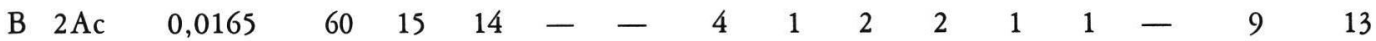

$\begin{array}{lllllllllllllllll}\text { В } & 2 \mathrm{Bc} & 0,0169 & 53 & 17 & 14 & 1 & - & 6 & 1 & 3 & 2 & 3 & \mathrm{x} & - & 10 & 12\end{array}$

$\begin{array}{lllllllllllllllllll}\text { B } & 1 \text { Ac } & 0,0409 & 65 & 16 & 2 & \mathrm{x} & - & 5 & \mathbf{x} & 5 & 1 & 3 & 1 & - & 8 & 16\end{array}$

$\begin{array}{llllllllllllllllll}\text { B } & 1 \mathrm{Bc} & 0,0351 & 64 & 13 & 6 & 1 & - & 6 & 2 & 3 & 2 & 2 & \mathrm{x} & - & 9 & 15\end{array}$ 
Tab. 2: Schwermineralanalysen in der Fraktion 0,063 - 0,1 mm für Profil B auf der Basis von 100 transparenten Körnern.
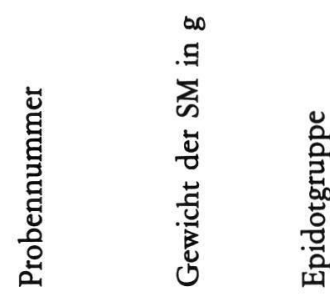

relative Korn \% der transparenten Schwermineralkörner
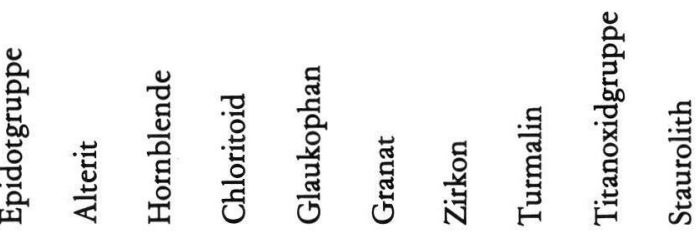

relative Korn \% aller gezählten

Körner
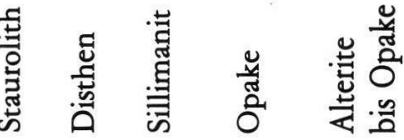

\begin{tabular}{|c|c|c|c|c|c|c|c|c|c|c|c|c|c|c|c|c|}
\hline \multicolumn{2}{|c|}{ B $10 A b$} & \multirow{2}{*}{$\begin{array}{l}0,0031 \\
0,0029\end{array}$} & \multirow{2}{*}{$\begin{array}{l}62 \\
59\end{array}$} & \multirow{2}{*}{$\begin{array}{r}11 \\
8\end{array}$} & \multirow{2}{*}{$\begin{array}{l}2 \\
8\end{array}$} & \multirow{2}{*}{$\begin{array}{l}- \\
-\end{array}$} & \multirow{2}{*}{$\begin{array}{c}- \\
1\end{array}$} & \multirow{2}{*}{$\begin{array}{l}2 \\
3\end{array}$} & \multirow{2}{*}{$\begin{array}{r}10 \\
8\end{array}$} & \multirow[t]{2}{*}{$\cdot 7$} & \multirow[b]{2}{*}{4} & \multirow[t]{2}{*}{1} & \multirow{2}{*}{$\begin{array}{r}- \\
1\end{array}$} & \multirow{2}{*}{ - } & 6 & \multirow{2}{*}{$\begin{array}{l}20 \\
13\end{array}$} \\
\hline \multicolumn{2}{|c|}{ B $10 \mathrm{Bb}$} & & & & & & & & & & & & & & & \\
\hline \multicolumn{2}{|c|}{$\mathrm{B} 9 \mathrm{Ab}$} & 0,0080 & 70 & 3 & 1 & - & - & - & 16 & 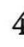 & & 1 & - & - & & \\
\hline 3 & $9 \mathrm{Bb}$ & 0,0098 & 53 & 6 & 3 & - & - & - & 28 & & & 2 & 1 & - & 15 & \\
\hline & $8 \mathrm{Ab}$ & 0,0018 & 51 & 5 & 10 & - & 1 & 2 & 18 & 3 & & 3 & - & - & & \\
\hline 3 & $8 \mathrm{Bb}$ & 0,0011 & 51 & 8 & 13 & - & - & 4 & 15 & J & & 1 & - & - & & \\
\hline & $7 \mathrm{Ab}$ & 0,0031 & 60 & 7 & 10 & - & - & 6 & 6 & 3 & & & 2 & - & & \\
\hline 3 & $7 \mathrm{Bb}$ & 0,0012 & 57 & 10 & 8 & - & - & 3 & 10 & 0 & & 2 & - & - & 0 & \\
\hline & $6 \mathrm{Ab}$ & 0,0052 & 64 & 5 & 5 & - & - & 3 & 11 & & & & - & - & & \\
\hline 3 & $6 \mathrm{Bb}$ & 0,0059 & 66 & 14 & 3 & - & - & 3 & 9 & 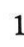 & & , & - & - & 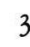 & \\
\hline & $5 \mathrm{Ab}$ & 0,0282 & 62 & 8 & 5 & - & - & 3 & 7 & j & & 4 & - & - & & \\
\hline & $5 \mathrm{Bb}$ & 0,0237 & 65 & 11 & 4 & - & - & 3 & 6 & , & & & 1 & - & & \\
\hline & $4 \mathrm{Ab}$ & 0,0065 & 53 & 16 & 5 & - & - & 6 & 7 & 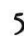 & & & 2 & - & & \\
\hline B & $4 \mathrm{Bb}$ & 0,0089 & 53 & 12 & 11 & - & - & 6 & 12 & 3 & & 1 & - & - & & \\
\hline & $3 \mathrm{Ab}$ & 0,0093 & 64 & 14 & 5 & - & - & 3 & 5 & 4 & 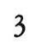 & & 1 & 1 & & \\
\hline B & $3 \mathrm{Bb}$ & 0,0076 & 60 & 14 & 3 & - & - & 2 & 11 & 4 & & 7 & - & - & & \\
\hline B & $2 \mathrm{Ab}$ & 0,0050 & 66 & 6 & 11 & - & - & 4 & 8 & 1 & ? & 1 & - & - & & \\
\hline B & $2 \mathrm{Bb}$ & 0,0059 & 58 & 9 & 19 & - & - & 4 & 3 & 1 & & J & 1 & - & 2 & \\
\hline 3 & $1 \mathrm{Ab}$ & 0,0110 & 48 & 13 & 12 & - & - & 10 & 8 & 4 & 4 & 1 & - & - & 6 & \\
\hline B & $1 \mathrm{Bb}$ & 0,0116 & 50 & 14 & 13 & - & - & 10 & 5 & 3 & 3 & 1 & 1 & - & 4 & \\
\hline
\end{tabular}


Tab. 3: Schwermineralanalysen in der Fraktion 0,2-0,4 mm für Profil B auf der Basis von 100 transparenten Körnern.

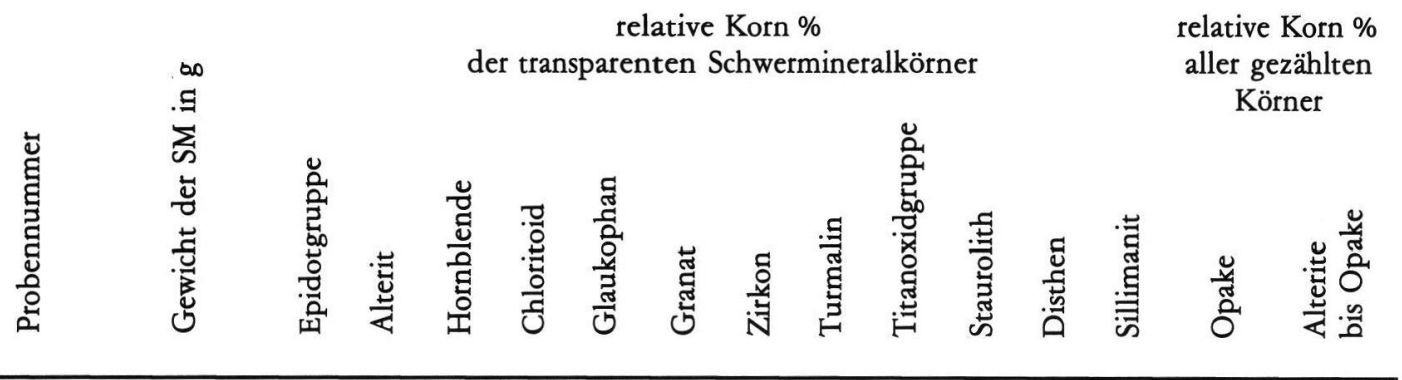

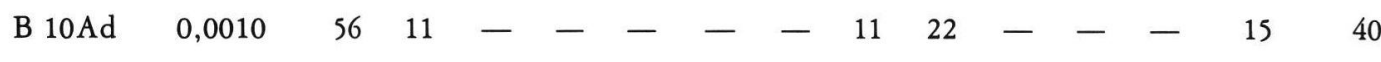

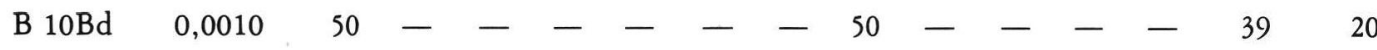

B 9Ad $0,0036 \quad 138$ - 8 - $\quad$ - $39-373$ - 37 24

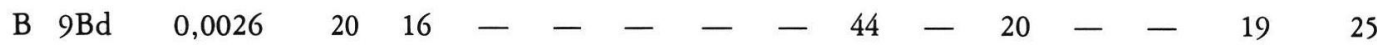

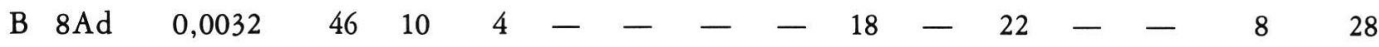

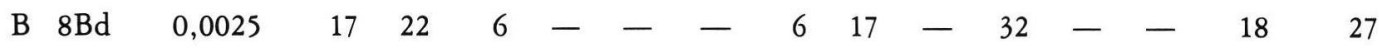

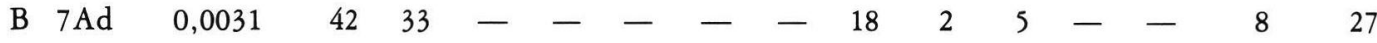

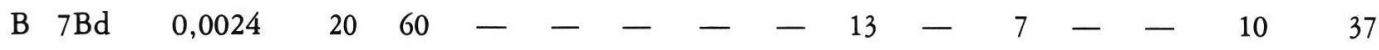

B 6Ad $0,0017 \quad 1362 \quad 6-6-13-6-10 \quad 30$

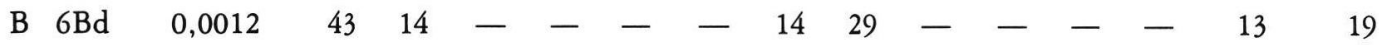

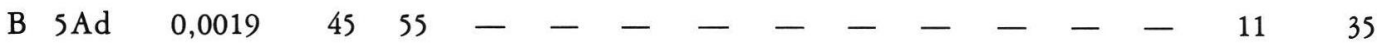

B 5 Bd $0,0020 \quad 35 \quad 47 \quad 6 \quad-\quad-\quad 12-6-48$

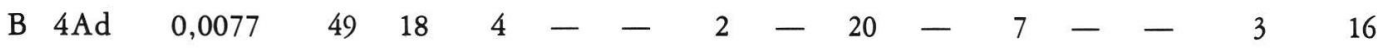

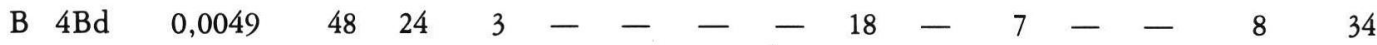

$\begin{array}{llllllllllllllll} & \text { B } 3 \text { Ad } & 0,0183 & 36 & 12 & 22 & - & - & 2 & 2 & 16 & - & 10 & - & 4 & 16\end{array}$

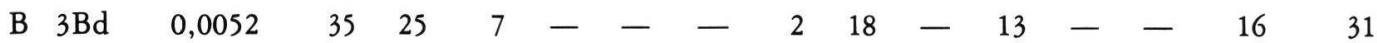

$\begin{array}{llllllllllllllllll}\text { B } & 2 \text { Ad } & 0,0061 & 18 & 14 & 12 & - & - & 10 & 11 & 19 & 4 & 12 & - & - & 14 & 20\end{array}$

$\begin{array}{llllllllllllllllll}\mathrm{B} & 2 \mathrm{Bd} & 0,0039 & 25 & 12 & 18 & - & 1 & 5 & 3 & 21 & 3 & 12 & - & - & 17 & 24\end{array}$

$\begin{array}{llllllllllllllllll}\text { B } & 1 \text { Ad } & 0,0044 & 36 & 27 & 12 & - & - & 1 & 1 & 12 & 5 & 6 & - & - & 11 & 28\end{array}$

$\begin{array}{lllllllllllllllll}\mathrm{B} & 1 \mathrm{Bd} & 0,0041 & 12 & 38 & - & - & - & 8 & 4 & 15 & - & 15 & 8 & & \end{array}$ 
Tab. 4: Schwermineralanalysen in der Fraktion 0,063-0,4 mm für Profil B auf der Basis von 100 transparenten Körnern.

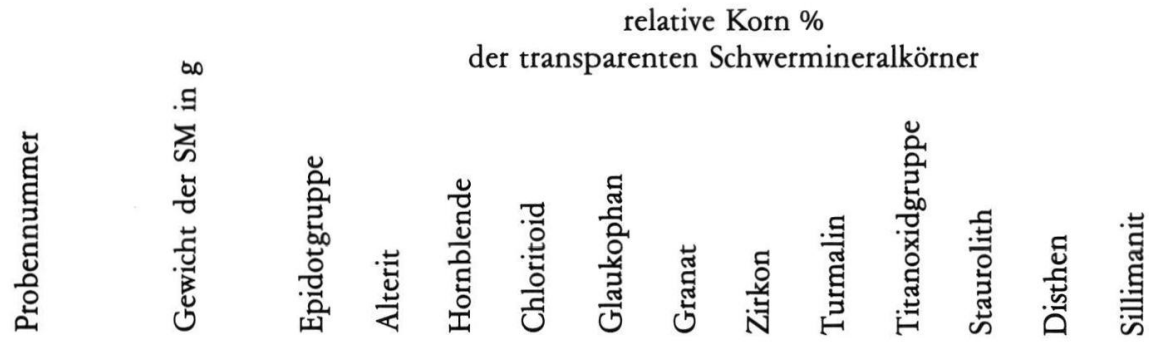

relative Korn \% aller gezählten Körner

\begin{tabular}{|c|c|c|c|c|c|c|c|c|c|c|c|c|c|c|c|c|}
\hline \multicolumn{2}{|c|}{ B $10 \mathrm{Aa}$} & \multirow{2}{*}{$\begin{array}{l}0,0010 \\
0,0009\end{array}$} & \multirow{2}{*}{$\begin{array}{l}58 \\
50\end{array}$} & \multirow{2}{*}{$\begin{array}{l}14 \\
33\end{array}$} & \multirow{2}{*}{$\begin{array}{r}3 \\
17\end{array}$} & \multirow{2}{*}{-} & \multirow{2}{*}{ - } & \multirow{2}{*}{-} & \multirow{2}{*}{-} & \multirow{2}{*}{$\begin{array}{l}22 \\
-\end{array}$} & \multirow{2}{*}{-} & \multirow{2}{*}{$\begin{array}{r}3 \\
-\end{array}$} & \multirow{2}{*}{-} & \multirow{2}{*}{-} & \multirow[b]{2}{*}{10} & \multirow{2}{*}{$\begin{array}{l}30 \\
35\end{array}$} \\
\hline \multicolumn{2}{|c|}{$\mathrm{B} 10 \mathrm{Ba}$} & & & & & & & & & & & & & & & \\
\hline B & $9 \mathrm{Aa}$ & 0,0044 & 52 & 12 & 1 & - & - & 1 & - & 24 & 2 & 7 & 1 & - & 11 & 31 \\
\hline \multicolumn{2}{|c|}{$\mathrm{B} 9 \mathrm{Ba}$} & 0,0060 & 52 & 3 & 1 & - & 1 & 1 & 9 & 17 & 7 & 7 & 2 & - & 14 & 22 \\
\hline \multirow{2}{*}{\multicolumn{2}{|c|}{$\begin{array}{l}\mathrm{B} 8 \mathrm{Aa} \\
\mathrm{B} 8 \mathrm{Ba}\end{array}$}} & 0,0059 & 25 & 42 & 8 & - & - & - & 8 & 17 & - & - & - & - & 3 & 10 \\
\hline & & 0,0043 & 54 & 12 & 10 & - & - & 2 & 8 & 3 & 1 & 8 & 1 & 1 & 14 & 21 \\
\hline \multicolumn{2}{|r|}{$7 \mathrm{Aa}$} & 0,0024 & 43 & 19 & 13 & - & - & - & - & 13 & 2 & - & 7 & - & 14 & 25 \\
\hline B & $7 \mathrm{Ba}$ & 0,0021 & 60 & 13 & 4 & - & - & - & - & 17 & - & 6 & - & - & 9 & 30 \\
\hline 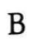 & $6 \mathrm{Aa}$ & 0,0033 & 59 & 17 & 6 & - & 1 & 2 & - & 7 & 2 & 3 & 2 & - & 6 & 26 \\
\hline B & $6 \mathrm{Ba}$ & 0,0028 & 52 & 15 & 8 & - & - & 1 & 4 & 8 & 1 & 5 & 6 & - & 5 & 20 \\
\hline B & $5 \mathrm{Aa}$ & 0,0071 & 51 & 31 & 6 & - & - & 1 & 1 & 7 & - & 1 & 1 & - & 8 & 23 \\
\hline B & $5 \mathrm{Ba}$ & 0,0065 & 64 & 16 & 5 & - & - & 1 & 5 & 3 & 3 & 2 & 1 & - & 6 & 19 \\
\hline & $4 \mathrm{Aa}$ & 0,0080 & 49 & 18 & 8 & - & - & 2 & 3 & 11 & 4 & 3 & - & - & 5 & 23 \\
\hline & $4 \mathrm{Ba}$ & 0,0074 & 43 & 21 & 15 & - & - & 8 & 2 & 2 & 4 & 5 & - & - & 7 & 21 \\
\hline & $3 \mathrm{Aa}$ & 0,0067 & 51 & 20 & 7 & - & - & 5 & 7 & 5 & 1 & 2 & 2 & - & 8 & 13 \\
\hline B & $3 \mathrm{Ba}$ & 0,0065 & 57 & 19 & 6 & - & - & 6 & 2 & 4 & 3 & 1 & 1 & - & 3 & 23 \\
\hline & $2 \mathrm{Aa}$ & 0,0071 & 45 & 11 & 13 & - & - & 7 & 2 & 10 & 2 & 8 & 2 & - & 6 & 11 \\
\hline$D$ & $2 \mathrm{Ba}$ & 0,0046 & 41 & 16 & 15 & - & - & 5 & - & 11 & 3 & 9 & - & - & 2 & 19 \\
\hline & $1 \mathrm{Aa}$ & 0,0040 & 40 & 33 & 10 & - & 1 & 7 & - & 5 & - & 3 & 1 & - & 11 & 21 \\
\hline & $1 \mathrm{Ba}$ & 0,0047 & 40 & 20 & 13 & 1 & - & 2 & 2 & 15 & - & 5 & 2 & - & 8 & 31 \\
\hline
\end{tabular}

Die vollständigen Tabellen mit den Profilen A und C sind in der Dipl.-Arb. WyPYrSCZYK (1983) aufgeführt. Die Tabellen können bei den Autoren angefordert werden. 
Tab. 5: Mittelwerte und Standardabweichungen für den Schwermineralgehalte in der Fraktion 0,1-0,2 mm aus den Zählergebnissen der Profile A, B, C

\begin{tabular}{|c|c|c|c|c|c|c|c|c|c|c|c|c|c|c|c|c|c|c|c|c|}
\hline \multirow{2}{*}{$\begin{array}{l}\text { Mittelwerte } \\
\text { für die Proben }\end{array}$} & \multicolumn{2}{|c|}{$\begin{array}{c}\text { A } 10, \mathrm{~B} 10 \\
\text { C } 10\end{array}$} & \multicolumn{2}{|c|}{$\begin{array}{l}\text { A9, B9, } \\
\text { C9 }\end{array}$} & \multicolumn{2}{|c|}{$\begin{array}{l}\mathrm{A} 8, \mathrm{~B} 8 \\
\mathrm{C} 8\end{array}$} & \multicolumn{2}{|c|}{$\begin{array}{c}\text { A } 7, \text { B } 7 \\
\text { C } 7\end{array}$} & \multicolumn{2}{|c|}{$\begin{array}{c}\text { A6, B6 } \\
\text { C6 }\end{array}$} & \multicolumn{2}{|c|}{ A 5, B 5} & \multicolumn{2}{|c|}{$\begin{array}{l}\text { A } 4, \mathrm{~B} 4 \\
\mathrm{C} 4\end{array}$} & \multicolumn{2}{|c|}{$\mathrm{B} 3, \mathrm{C} 3$} & \multicolumn{2}{|c|}{$\mathrm{B} 2, \mathrm{C} 2$} & \multicolumn{2}{|c|}{$\mathrm{B} 1, \mathrm{C} 1$} \\
\hline & $\overline{\mathbf{x}}$ & $\delta$ & $\overline{\mathbf{x}}$ & $\delta$ & $\overline{\mathbf{x}}$ & $\delta$ & $\overline{\mathbf{x}}$ & $\delta$ & $\overline{\mathbf{x}}$ & $\delta$ & $\overline{\mathbf{x}}$ & $\delta$ & $\overline{\mathbf{x}}$ & $\delta$ & $\overline{\mathbf{x}}$ & $\delta$ & $\overline{\mathbf{x}}$ & $\delta$ & $\overline{\mathbf{x}}$ & $\delta$ \\
\hline Epidotgruppe & 66,5 & 6,8 & 63,5 & 10,1 & 64,8 & 6,1 & 63,7 & 10,3 & 64,0 & 5,3 & 57,8 & 10,2 & 60,5 & 3,3 & 59,0 & 3,7 & 51,5 & 6,4 & 55,5 & 1,3 \\
\hline Hornblende & 1,7 & 1,6 & 2,3 & 1,6 & 2,2 & 2,5 & 4,2 & 3,2 & 4,0 & 2,1 & 1,5 & 0,6 & 3,2 & 2,0 & 5,5 & 0,6 & 11,5 & 3,1 & 7,0 & 4,2 \\
\hline Chloritoid & 0,2 & 0,4 & 0,5 & 0,8 & 0,3 & 0,5 & - & - & 0,3 & 0,8 & 0,5 & 1,0 & 0,3 & 0,5 & 1,3 & 1,3 & 0,3 & 0,5 & 0,8 & 1,0 \\
\hline Glaukophan & - & - & - & - & - & - & 0,2 & 0,4 & - & - & - & - & - & - & - & - & 0,3 & 0,5 & - & - \\
\hline Granat & 0,7 & 0,5 & 0,7 & 0,8 & 2,8 & 1,3 & 2,8 & 2,5 & 3,2 & 2,3 & 1,0 & 0,8 & 8,2 & 1,7 & 8,5 & 3,5 & 6,5 & 2,4 & 7,8 & 2,1 \\
\hline Zirkon & 2,7 & 2,1 & 3,0 & 1,7 & 1,5 & 1,2 & 1,7 & 1,0 & 2,8 & 1,5 & 1,8 & 1,0 & 3,3 & 2,3 & 3,3 & 1,7 & 2,0 & 0,8 & 2,8 & 1,5 \\
\hline Turmalin & 6,3 & 2,4 & 11,0 & 5,8 & 5,2 & 0,8 & 4,7 & 1,8 & 4,3 & 1,5 & 3,0 & 1,4 & 3,3 & 1,9 & 2,5 & 1,3 & 2,8 & 2,1 & 3,8 & 1,5 \\
\hline Titanoxidgruppe & 3,8 & 2,6 & 2,7 & 1,8 & 3,0 & 1,7 & 1,5 & 1,1 & 2,5 & 2,0 & 0,8 & 1,0 & 3,0 & 2,1 & 2,3 & 0,5 & 2,3 & 1,3 & 2,5 & 1,7 \\
\hline Staurolith & 7,2 & 1,9 & 6,0 & 1,6 & 3,5 & 1,2 & 2,3 & 1,5 & 3,2 & 1,7 & 3,8 & 1,0 & 2,3 & 1,7 & 2,8 & 2,8 & 3,0 & 2,2 & 0,8 & 1,0 \\
\hline Disthen & 1,0 & 1,1 & 1,5 & 1,4 & 0,2 & 0,4 & 2,0 & 1,1 & 0,7 & 0,8 & 1,0 & 0,8 & 0,7 & 1,2 & 0,8 & 1,0 & 1,3 & 1,0 & 0,8 & 1,0 \\
\hline Sillimanit & - & - & - & - & - & - & 0,2 & 0,4 & - & - & 0,3 & 0,5 & 0,2 & 0,4 & - & - & - & - & 0,3 & 0,5 \\
\hline $\begin{array}{l}\text { Hornblende + } \\
\text { Granat }\end{array}$ & 2,5 & 1,4 & 4,5 & 1,1 & 5,3 & 2,9 & 7,2 & 5,0 & 7,5 & 1,6 & 2,8 & 1,7 & 11,7 & 2,9 & 15,3 & 3,9 & 18,5 & 3,3 & 15,5 & 6,8 \\
\hline Stabile & 12,8 & 5,1 & 16,7 & 6,7 & 9,7 & 2,3 & 7,8 & 1,8 & 9,7 & 2,5 & 8,0 & 7,2 & 9,7 & 4,5 & 8,0 & 2,0 & 7,0 & 2,2 & 9,0 & 1,4 \\
\hline Metamorphe & 8,2 & 2,2 & 7,5 & 1,8 & 3,7 & 1,4 & 5,5 & 2,7 & 3,8 & 1,8 & 5,0 & 0,8 & 3,2 & 1,2 & 3,5 & 2,4 & 4,3 & 1,3 & 1,8 & 1,7 \\
\hline
\end{tabular}


Tab. 6: Schwermineralgehalt in der Fraktion $0,1-0,2 \mathrm{~mm}$ in $\mathrm{g}$ und Gew. \%.

\begin{tabular}{|c|c|c|c|c|c|c|c|c|c|}
\hline Probe & g & $\%$ & Probe & $\mathrm{g}$ & $\%$ & Probe & g & $\%$ & Mittelwert \\
\hline A10Ac & 0,0247 & 1,8687 & B10Ac & 0,0099 & 0,6654 & $\mathrm{C} 10 \mathrm{Ac}$ & 0,0223 & 2,1697 & \multirow{2}{*}{$1,5871 \pm 0,6708$} \\
\hline $\mathrm{A} 10 \mathrm{Bc}$ & 0,0272 & 1,8905 & $\mathrm{~B} 10 \mathrm{Bc}$ & 0,0133 & 0,8085 & $\mathrm{C} 10 \mathrm{Bc}$ & 0,0240 & 2,1196 & \\
\hline A 9 Ac & 0,0051 & 0,3002 & B 9Ac & 0,0155 & 0,8470 & $\mathrm{C} 9 \mathrm{Ac}$ & 0,0243 & 1,2932 & \multirow{2}{*}{$0,7761 \pm 0,4153$} \\
\hline $\mathrm{A} 9 \mathrm{Bc}$ & 0,0047 & 0,2694 & $\mathrm{~B} 9 \mathrm{Bc}$ & 0,0160 & 0,8527 & $\mathrm{C} 9 \mathrm{Bc}$ & 0,0223 & 1,0939 & \\
\hline A $8 \mathrm{Ac}$ & 0,0155 & 0,9096 & B 8 Ac & 0,0067 & 0,3637 & C $8 \mathrm{Ac}$ & 0,0263 & 1,3545 & \multirow{2}{*}{$0,9672 \pm 0,3489$} \\
\hline $\mathrm{A} 8 \mathrm{Bc}$ & 0,0148 & 0,8592 & B $8 B c$ & 0,0087 & 1,1008 & C $8 \mathrm{Bc}$ & 0,0235 & 1,2151 & \\
\hline A $7 \mathrm{Ac}$ & 0,0378 & 2,0568 & B 7 Ac & 0,0395 & 2,3421 & C $7 \mathrm{Ac}$ & 0,0078 & 0,4162 & \multirow{2}{*}{$1,5178 \pm 0,8951$} \\
\hline $\mathrm{A} 7 \mathrm{Bc}$ & 0,0330 & 1,9021 & $\mathrm{~B} 7 \mathrm{Bc}$ & 0,0408 & 2,0511 & $\mathrm{C} 7 \mathrm{Bc}$ & 0,0066 & 0,3386 & \\
\hline A 6 Ac & 0,0052 & 0,2781 & B 6 Ac & 0,0080 & 0,4359 & C 6 Ac & 0,0367 & 2,1907 & \multirow{2}{*}{$1,0429 \pm 1,0556$} \\
\hline A $6 \mathrm{Bc}$ & 0,0042 & 0,2410 & B $6 \mathrm{Bc}$ & 0,0097 & 0,5232 & C $6 \mathrm{Bc}$ & 0,0460 & 2,5882 & \\
\hline A $5 \mathrm{Ac}$ & 0,0066 & 0,3363 & B $5 \mathrm{Ac}$ & 0,0162 & 0,8702 & & & & \multirow{2}{*}{$0,6334 \pm 0,2223$} \\
\hline A $5 \mathrm{Bc}$ & 0,0134 & 0,6310 & B $5 \mathrm{Bc}$ & 0,0128 & 0,6961 & & & & \\
\hline A 4 Ac & 0,0310 & 1,6268 & B $4 \mathrm{Ac}$ & 0,0290 & 1,5805 & C $4 \mathrm{Ac}$ & 0,0224 & 1,4025 & \multirow{2}{*}{$1,3254 \pm 0,4844$} \\
\hline A $4 \mathrm{Bc}$ & 0,0293 & 1,6570 & B $4 \mathrm{Bc}$ & 0,0067 & 0,3762 & $\mathrm{C} 4 \mathrm{Bc}$ & 0,0213 & 1,3093 & \\
\hline & & & B 3 Ac & 0,0277 & 1,5958 & C $3 \mathrm{Ac}$ & 0,0179 & 0,9922 & \multirow{2}{*}{$1,3229 \pm 0,3580$} \\
\hline & & & $\mathrm{B} 3 \mathrm{Bc}$ & 0,0280 & 1,6673 & C $3 \mathrm{Bc}$ & 0,0180 & 1,0363 & \\
\hline & & & B $2 A c$ & 0,0165 & 0,9773 & C $2 \mathrm{Ac}$ & 0,0227 & 1,3559 & \multirow{2}{*}{$1,2143 \pm 0,2540$} \\
\hline & & & B $2 \mathrm{Bc}$ & 0,0169 & 1,0246 & C $2 \mathrm{Bc}$ & 0,0284 & 1,4995 & \\
\hline & & & B 1 Ac & 0,0409 & 2,2726 & C $1 \mathrm{Ac}$ & 0,0249 & 1,6233 & \multirow{2}{*}{$1,8595 \pm 0,3023$} \\
\hline & & & $\mathrm{B} 1 \mathrm{Bc}$ & 0,0351 & 1,8975 & $\mathrm{C} 1 \mathrm{Bc}$ & 0,0237 & 1,6445 & \\
\hline
\end{tabular}

